\title{
Small in-frame deletions and missense mutations in CADASIL: 3D models predict misfolding of Notch3 EGF-like repeat domains
}

\author{
Martin Dichgans ${ }^{1}$, Harald Ludwig ${ }^{1}$, Josef Müller-Höcker ${ }^{2}$, Albrecht Messerschmidt ${ }^{3}$ and \\ Thomas Gasser $^{1}$
}

\author{
${ }^{1}$ Department of N eurology, Klinikum Grosshadern; ${ }^{2}$ D epartment of Pathology; Ludwig-Maximilians-University, \\ Munich; ${ }^{3} \mathrm{M}$ ax-Planck-Institut für Biochemie, Abteilung Strukturforschung, Martinsried, Germany
}

\begin{abstract}
CADASIL (cerebral autosomal dominant arteriopathy with subcortical infarcts and leukoencephalopathy) is a hereditary microangiopathic condition causing stroke in young adults. The responsible gene has recently been identified as the Notch 3 gene. Notch 3 encodes a large transmembrane receptor with 34 extracellularly localised epidermal growth factor-like (EGF) repeat domains. We screened 71 unrelated CADASIL families for mutations in two exons coding for the first five EGF-like repeats and found mutations in $70 \%$ of the families $(n=50)$. Two types of mutations were identified: 48 families $(96 \%)$ had missense mutations and two families $(4 \%)$ had small in-frame deletions. Seven mutations occurred multiple times. All of them are C to $\mathrm{T}$ transitions that affect CpG dinucleotides, suggesting that their multiple occurrence is due to the hypermutability of this sequence. All mutations, including the two deletions, result in the gain or loss of a cysteine residue, thus substantiating the pivotal role of an uneven number of cysteine residues within EGF-like repeat domains of Notch3 in the pathogenesis of CADASIL. To study the potential effects of these mutations 3D homology models of the first six EGF domains were generated on the basis of NMR data from human fibrillin-1. These models predict domain misfolding for a subset of mutations. European Journal of Human Genetics (2000) 8, 280-285.
\end{abstract}

Keywords: CADASIL; Notch3; mutation; EGF-like repeat; 3D model

\section{Introduction}

CADASI L is an increasingly recognised autosomal dominant disorder leading to cerebrovascular manifestations in early adulthood. ${ }^{1}$ Affected individuals experience recurrent ischemic episodes with accumulating motor, sensory and cognitive deficits. ${ }^{2,3}$ The majority of patients eventually become demented and additional manifestations have been reported including migraine (30-40\%), psychiatric disturbance (30\%) and epileptic seizures (10\%). ${ }^{2,3}$ Brain magnetic resonance imaging (MRI) displays diffuse white matter abnormalities and small cystic lesions suggestive of small infarcts. ${ }^{4}$ The penetrance of MRI abnormalities in CADASIL is complete by

Correspondence: Dr M Dichgans, Department of Neurology, Klinikum Grosshadern, Ludwig-Maximilians-University, Marchioninstr. 15, D-81377 Munich, Germany. Tel: +4989 7095 3673; Fax:

+4989 7095 3677; E-mail: mdichgans@brain.nefo.med.uni-muenchen.de Received 10 September 1999; revised 10 December 1999; accepted 21 December 1999 age $35 .^{2}$ However, symptom onset varies considerably (age 14-66 years). ${ }^{3}$ The underlying pathology is mediated by a unique non-amyloid angiopathy involving small arteries $(100-400 \mu \mathrm{m})$ and capillaries, particularly in the brain but also in other organs. ${ }^{5}$

Recently, the Notch3 gene on chromosome19p13 was identified as the CADASIL disease gene. ${ }^{6} \mathrm{~N}$ otch genes encode evolutionary conserved cell surface receptors that regulate cell fate choices in vertebrates and invertebrates during embryonic development. ${ }^{7}$ This regulation occurs by interaction of Notch gene products with their transmembrane ligands on neighbouring cells. ${ }^{7,8}$

A striking feature of both the Notch receptors and their ligands is a large number of tandemly arranged epidermal growth factor-like (EGF-like) repeat domains which account for most of the extracellular domains of these proteins. ${ }^{7}$ EGFlike repeats have been identified in numerous extracellular and membrane bound proteins. ${ }^{9,10}$ Their classification is 
based mainly on the spacing and presence of six cysteine residues within approximately 40 aminoacid residues. The structures of a number of EGF-like domains have been determined, and most of them are consistent with an EGFlike disulfide bonding pattern. ${ }^{10-16}$

On screening 50 CADASIL families for mutations along the entire coding sequence of the Notch 3 gene, Joutel et al found mutations in $90 \%$ of the families. ${ }^{17}$ All mutations were located in one of the 34EGF-like repeat domains with a strong cluster in two exons coding for the first five repeats. All mutations were missen se mutations predicted to result in a loss or a gain of cysteine residue. Based on this observation the authors hypothesised that aberrant dimerisation of Notch3, due to abnormal disulfide bridging with another Notch 3 molecule or with another protein, may be involved in the pathogenesis of the disorder. ${ }^{17}$

In the current study we performed mutational analysis in 71 unrelated CADASI L families by focusing on exons 3 and 4 which code for the first five EGF-like repeats of the Notch3 gene product. 3D homology models were generated to study the potential effects of the observed mutations.

\section{Patients and methods \\ Patients}

We studied 71 index cases and their affected relatives from 71 unrelated Caucasian families (61 German, 4 Swiss, 3 Austrian, and 3 British). Probands were selected on the basis of

(1) A clinical syndrome of recurrent ischemic episodes, cognitive deterioration, migraine with aura, psychiatric disturbance, or a combination of these features;

(2) A cerebral MRI showing microangiopathic changes, and

(3) A family history consistent with autosomal dominant inheritance.

In all families the diagnosis of CADASI L had been confirmed by biopsy ( $n=66$ ) or autopsy $(n=5){ }^{18,19}$

\section{Mutation analysis}

Following informed consent, genomic DNA was isolated from peripheral blood leukocytes. Exons 3 and 4 of the $\mathrm{N}$ otch 3 gene were amplified using the following intronic primers: exon 3 (F: 5'TGTGCTGCCCAACCAAGCCA; R: 5'ACTGACCACACCCCCGACTA);

exon 4 (F: 5'TAGTCGGGGGTGTGGTCAGT; R: 5'CCTCTGACTCTCCTGAGTAG). ${ }^{17}$

PCR conditions were as follows: initial denaturation $5 \mathrm{~min}$ at $94^{\circ} \mathrm{C}$, followed by 35 cycles at $94^{\circ} \mathrm{C}$ for 30 s (denaturation), $65^{\circ} \mathrm{C}$ for $30 \mathrm{~s}$ (annealing) and $72^{\circ} \mathrm{C}$ for $30 \mathrm{~s}$ (extension), with a $7 \mathrm{~min}$ final extension at $72^{\circ} \mathrm{C}$. PCR products were subjected to direct automated sequencing using dideoxy-terminator cycle sequencing (BigDye ${ }^{\mathrm{TM}}$ sequencing Ready Reaction Kit, Perkin-Elmer, Foster City, CA) and an Applied Biosystems model 377 automated sequencer (Applied Biosystems division, Perkin-Elmer Corporation, Foster City, CA). All products were sequenced on both strands. The human Notch 3 cDNA and human Notch3 protein sequences were taken from GenBank (accession no. U97669) and a published paper. $^{17}$

\section{D Modelling}

3D modelling was done on the basis of the NMR structure of the 32nd and 33rd calcium binding (cb) EGF-like domain pair of human fibrillin- $1^{16}$ and a multiple aminoacid sequence alignment of this cbEGF-like domain pair with EGF-like domain pairs 1-2, 3-4 and 5-6 from human Notch 3 (Table1). Alignment was arranged manually with the disulfide bridge forming cysteines as anchor points. The alignment of domain pairs was chosen, since Notch 3 domains 1,3 and 5 align well on the 32nd EGF-like domain of human fibrillin-1 and Notch 3 domains2, 4 and 6 align well on the 33rd EGF-like domain of human fibrillin-1 (Table1). Models are presented as single domains because of uncertainties regarding interdomain arrangement.

Model building was done with program ' $\mathrm{O}$ '. ${ }^{20}$ Mutated side chains were put into energetically favourable conformations with option 'lego-side-chain'. Those parts of the models where insertions or deletions had to be introduced were regularised with option 'refi-zone'. Energy minimisation of the models was not carried out because it did not promise further improvement of the models.

\section{Results}

\section{Mutation detection}

Seventy-one unrelated CADASIL families were analysed for mutations in the cluster region (exons 3 and 4 ) of the $\mathrm{N}$ otch 3

Table 1 Multiple aminoacid sequence alignment between the 32nd and 33rd cbEGF-like domains from human fibrillin-1 and EGF-like domain pairs 1-2, 3-4 and 5-6 from Notch3

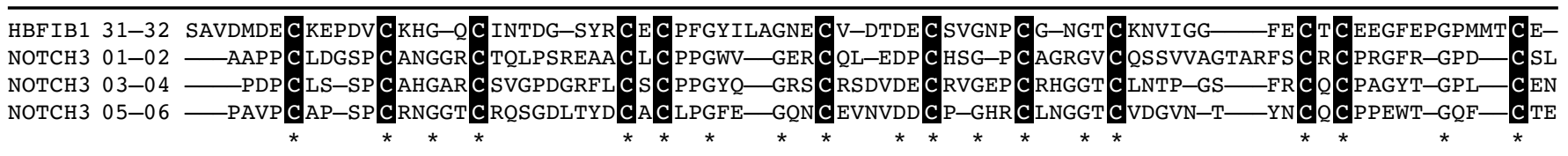

The alignment was done manually with the pattern of cysteines (black boxed) as anchor points. *Consensus residues. 
Table 2 Notch3 mutations in 50 CADASIL families

\begin{tabular}{|c|c|c|c|c|c|}
\hline No. of families & Notch3 nucleotide & Notch3 mutation & Amino acid exchange & Exon & Domain \\
\hline 6 & 346 & $\mathrm{CGT} \rightarrow \mathrm{TGT}$ & Arg90 $\rightarrow$ Cys & N3 & EGF 2 \\
\hline 1 & 356 & $\mathrm{TGC} \rightarrow \mathrm{TTC}$ & Cys93 $\rightarrow$ Phe** & N3 & EGF 2 \\
\hline 3 & 406 & $\mathrm{CGT} \rightarrow \mathrm{TGT}$ & Argl10 $\rightarrow$ Cys & N3 & EGF 2 \\
\hline 1 & 428 & $\mathrm{TGC} \rightarrow \mathrm{TTC}$ & Cysl17 $\rightarrow$ Phe & N4 & EGF 2 \\
\hline 1 & 445 & $\mathrm{TGC} \rightarrow \mathrm{TTC}$ & Cys123 $\rightarrow$ Phe* & N4 & EGF 3 \\
\hline 10 & 475 & $\mathrm{CGC} \rightarrow \mathrm{TGC}$ & Arg133 $\rightarrow$ Cys & N4 & EGF 3 \\
\hline 5 & 499 & $\mathrm{CGC} \rightarrow \mathrm{TGC}$ & Arg141 $\rightarrow$ Cys & N4 & EGF 3 \\
\hline 1 & 508 & $\mathrm{TGC} \rightarrow \mathrm{TCC}$ & Cys144 $\rightarrow$ Ser* & N4 & EGF 3 \\
\hline 1 & 508 & $\mathrm{TGC} \rightarrow \mathrm{TAC}$ & Cys144 $\rightarrow$ Tyr* & N4 & EGF 3 \\
\hline 1 & 525 & $\mathrm{TAC} \rightarrow \mathrm{TGC}$ & Tyr150 $\rightarrow$ Cys* & N4 & EGF 3 \\
\hline 2 & 535 & $\mathrm{CGC} \rightarrow \mathrm{TGC}$ & Arg153 $\rightarrow$ Cys & N4 & EGF 3 \\
\hline 5 & 583 & $\mathrm{CGC} \rightarrow \mathrm{TGC}$ & Arg169 $\rightarrow$ Cys & N4 & EGF 4 \\
\hline 1 & 599 & $\mathrm{TGC} \rightarrow \mathrm{TAC}$ & Cys174 $\rightarrow$ Tyr & N4 & EGF 4 \\
\hline 6 & 622 & $\mathrm{CGC} \rightarrow \mathrm{TGC}$ & Arg182 $\rightarrow$ Cys & N4 & EGF 4 \\
\hline 1 & 625 & $\mathrm{TGC} \rightarrow \mathrm{CGC}$ & Cys183 $\rightarrow$ Arg & N4 & EGF 4 \\
\hline 1 & 625 & $\mathrm{TGC} \rightarrow \mathrm{AGC}$ & Cys183 $\rightarrow$ Ser* & N4 & EGF 4 \\
\hline 1 & 631 & $\mathrm{TGT} \rightarrow \mathrm{CGT}$ & Cys185 $\rightarrow$ Arg & N4 & EGF 4 \\
\hline 1 & 658 & $\mathrm{TGT} \rightarrow \mathrm{TIT}$ & Cys194 $\rightarrow$ Phe* & N4 & EGF 4 \\
\hline 1 & $317-331$ & deletion & D80-S84del* & N4 & EGF 2 \\
\hline 1 & $537-545$ & deletion & R153-C155del* & N4 & EGF 3 \\
\hline
\end{tabular}

All mutations are predicted to involve cysteine residues. In 38 families the mutation is predicted to create a cysteine, whereas in 12 families th $\epsilon$ mutation is predicted to delete a cysteine residue. Nucleotide numbers are given based on the human Notch3 cDNA sequence taken from GenBank (accession no. U97669); *novel mutation.

gene by direct sequencing of genomic DNA. Mutations were found in 50 (70\%) of the families. Of the 20 different mutations identified 18 were missense mutations (90\%) and two were deletions (10\%). Nine mutations are novel (Table2).

Missense mutations All the missense mutations are predicted to involve cysteine residues, ie to replace the wild-type aminoacid with a cysteine residue (eight mutations) or to replace one of the cysteine residues with another aminoacid (ten mutations). Seven mutations were found more than once, ie 2-10 times. All of them are C to T transitions leading to the substitution of an arginine by a cysteine residue. Eleven mutations were found only once. Ten of them are predicted to replace a cysteine residue with another aminoacid.

Deletions We found one 15-bp and one 9-bp deletion predicted to result in the loss of five and three aminoacids, respectively. In both cases the deleted aminoacids include one cysteine residue. Both deletions occur at sites containing DNA repeat sequences. The 317-331del sequence is flanked by a $7 \mathrm{bp}-$ CCCCTGT repeat while the $537-545 \mathrm{del}$ sequence is flanked by a 4bp-GCCG repeat. In both cases one of the repeats is deleted by the mutation which is consistent with misalignment during DNA replication. ${ }^{21}$

Polymorphisms In addition to disease-causing mutations there were several silent nucleotide polymorphisms (Table3).

\section{Discussion}

Our study extends the spectrum of disease-causing mutations in CADASIL by adding small in-frame deletions. Both deletions are predicted to result in the loss of one cysteine residue. In conjunction with the missense mutations reported here and previously ${ }^{17,22,23}$ these deletions substantiate the pivotal role of an odd number of cysteine residues within EGF-like repeat domains of Notch3 in the pathogenesis of this disorder.

The mechanisms by which mutations in CADASIL become pathogenic are currently unknown. However, at least two possibilities have to be considered. The first is domain misfolding. As illustrated by the modelled 3D structures of the first six EGF-like repeat domains (Figure1) each domain is predicted to contain an $\mathrm{N}$-terminal two-stranded $\beta$-sheet followed by a second shorter sheet, or double hairpin at the $\mathrm{C}$-terminus. The six cysteines form three disulfide bonds which stabilise the two $\beta$-strand substructures. Some of the mutations are predicted to be incompatible with this folding pattern. This is obvious for the two deletions which destroy sequences involved into $\beta$-strand formation and domain stabilisation (Figure1). Domain misfolding may further be predicted for a group of missense mutations where large side chains of the replacing residues are predicted to interfere with adjacent parts of the native structure (Cys to Arg; Cys to

Table 3 Silent nucleotide polymorphisms in exons 3 and 4 of the N otch 3 gene

\begin{tabular}{lllll}
\hline $\begin{array}{l}\text { Notch3 } \\
\text { nucleotide }\end{array}$ & $\begin{array}{l}\text { Nucleotide } \\
\text { change }\end{array}$ & $\begin{array}{l}\text { Amino acid } \\
\text { exchange }\end{array}$ & Exon & Domain \\
\hline 360 & CAG $\rightarrow$ CAA & none & N3 & EGF 2 \\
381 & ACC $\rightarrow$ ACT & none & N3 & EGF 2 \\
423 & CCT $\rightarrow$ CCC & none & N4 & EGF 2 \\
546 & CGA $\rightarrow$ CGG & none & N4 & EGF 3 \\
684 & GCG $\rightarrow$ GCA & none & N4 & EGF 5 \\
\hline
\end{tabular}


a

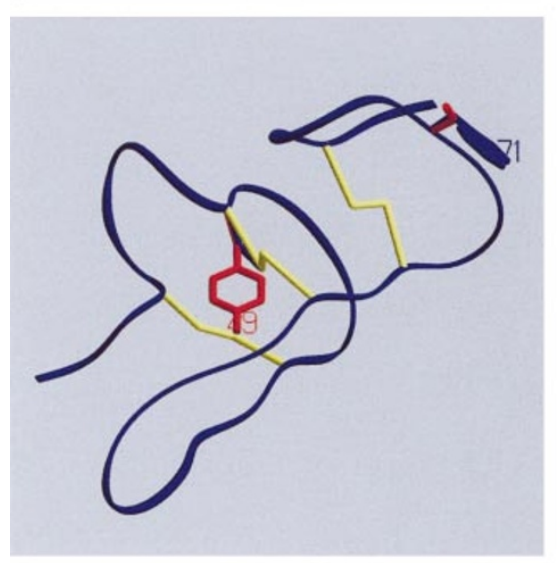

c

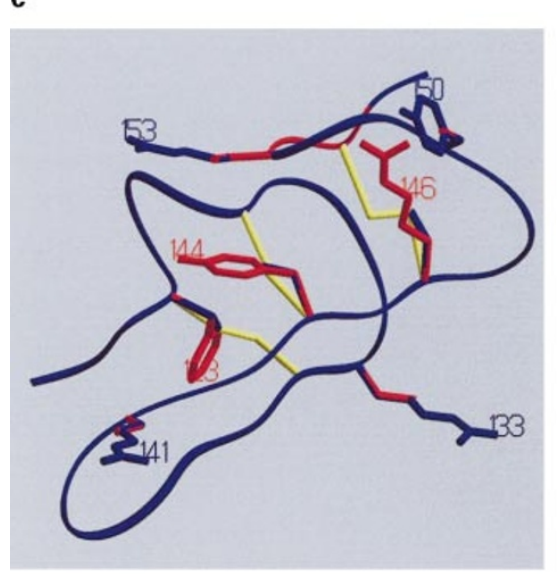

e

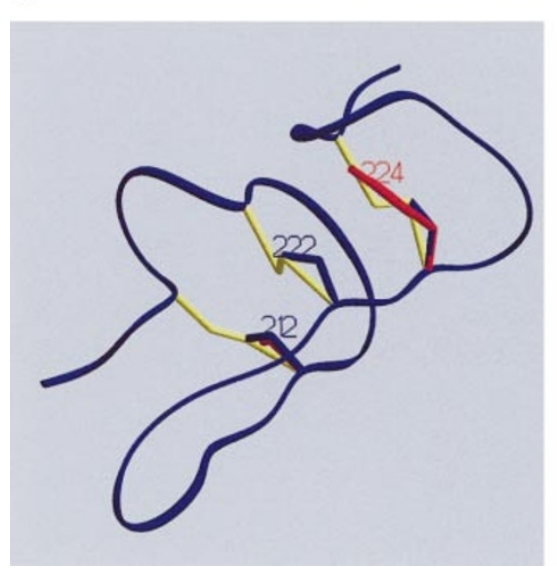

b

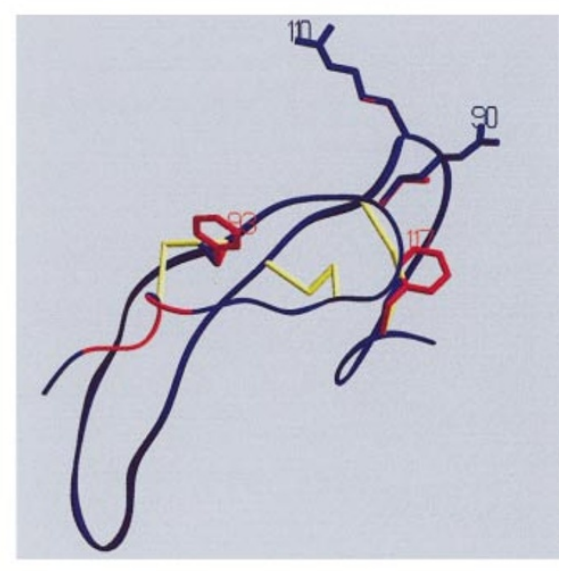

d

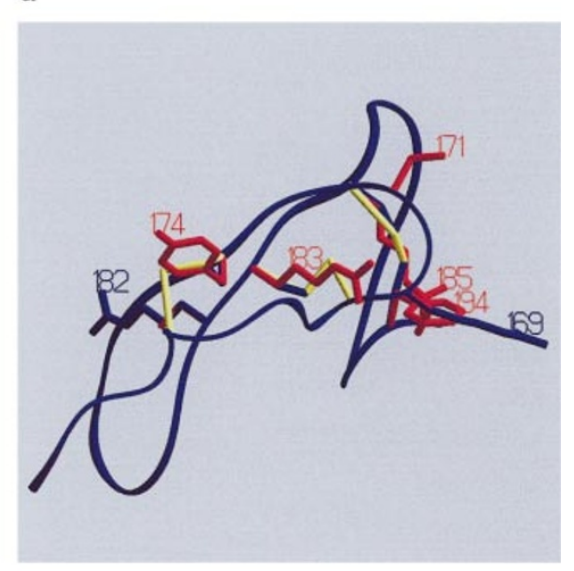

f

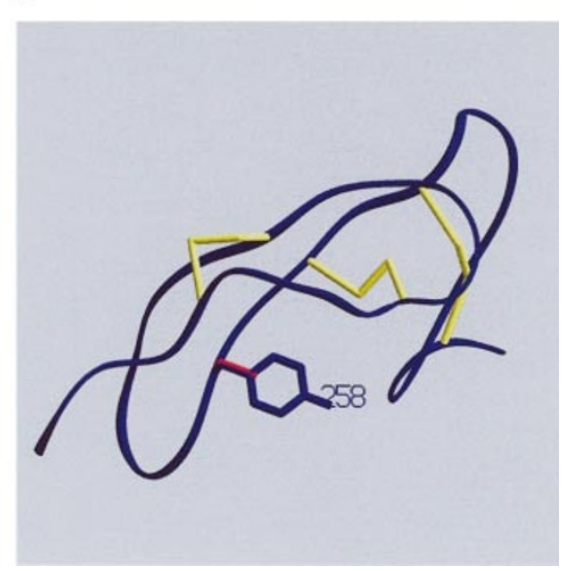

Figure $13 \mathrm{D}$ models of the predicted main chain traces of the first six Notch3 EGF-like repeat domains (a-f). The main chain traces are shown in blue (N-termini at the lower left). Each domain contains a two-stranded $\beta$-sheet followed by a second shorter sheet, or double hairpin. The six cysteines form three disulfide bonds (yellow). The two-stranded $\beta$-sheet is stabilised by two crossing disulfide bonds (1st to 3rd and 2nd to 4th cysteine residue), and the double hairpin is fixed by the 5th to 6th - disulfide bond. The 4th and 5 th cysteines are only one amino acid apart, so that the two $\beta$-strand substructures are closely connected. Wild-type side chains (blue) are shown at positions affected by missense mutations (mutated side chains in red). The two deletions are indicated as a red section within the main chain trace. For the purpose of completedness mutations identified in this study were combined with previously reported mutations: Cys49Tyr; Trp71Cys; Cys146Arg; Gly171Cys; Cys212Ser; Cys222Gly; Cys224Tyr; Tyr258Cys. ${ }^{17}$ This Figure was produced using SETOR. ${ }^{26}$ 
Phe; Cys to Tyr; Figure1, data not shown). In fact, it seems likely that all mutations substituting cysteine residues cause protein misfolding since these mutations disrupt disulfide bonds likely to be necessary for a correct fold of the respective domain (Figure1). The consequences of mutations generating new cysteine residues (Arg to Cys; Tyr to Cys; Trp to Cys; Gly to Cys) are difficult to predict. However, with an odd number of cysteines, new permutations of the bridging scheme might be favoured. This would also cause domain misfolding in these mutations.

Another possibility consists in the formation of intermolecular cross-links with other Notch 3 molecules (homodimers) or other cysteine-containing proteins (heterodimers). It is conceivable from Figure1, that mutations replacing an amino acid with a cysteine residue could provide the basis for such interactions in particular, since these mutations affect residues predicted to be situated on the surface of the molecule. Mutations that replace cysteine residues could have a similar effect by leaving an unpaired and thus reactive cysteine residue.

Seven mutations occurred multiple times. As previously shown, the multiple occurrence of mutations in CADASIL is not explained by founder effects. ${ }^{17}$ Interestingly, all the multiple occuring mutations found in this study and in the study by Joutel ${ }^{17}$ are $\mathrm{C}$ to $\mathrm{T}$ transitions affecting $\mathrm{CpG}$ dinucleotides. CPG dinucleotides have been shown to undergo germ-line transitions to TG and CA at frequencies six to seven times the base mutation rate. ${ }^{24}$ Thus we assume, that the multiple occurrence of these mutations may be attributed to the hypermutability of CPG dinucleotides, which involves methylation-mediated deamination of cytosines. $^{24}$

The reasons for the strong clustering of CADASIL mutations within exons coding for $\mathrm{N}$-terminal EGF repeats are still unknown. A comparison of exons 3 and 4 to more downstream coding sequences of $\mathrm{N}$ otch 3 failed to reveal a significant increase in GC content or in the frequency of CpG dinucleotides (results not shown). Thus a simple DNA-based explanation for the clustering of mutations is lacking. It has al ready been pointed out that the $\mathrm{N}$-terminal repeats are less conserved in between Notch 3 and otherwise highly homologous Notch proteins. ${ }^{17}$ In fact, sequence alignment with other Notch proteins revealed that Notch 3 lacks a region corresponding to parts of EGF-repeats 2 and 3 in the homologous proteins ${ }^{25}$ (own results, data not shown). The loss of this region is equivalent to a net loss of one EGF-repeat. Interestingly, the second Notch 3 EGF-repeat domain differs from most other Notch 3 EGF domains by an additional three to four aminoacid residues that are located in between the third and fourth cysteine residue. As indicated in Figure $1 b$ this is predicted to result in a longer two-stranded $\beta$-sheet, elongated in the direction of the $\mathrm{N}$-terminus. It is conceivable, that this could affect the interdomain arrangement in this region. However, whether structural features relate to the clustering of mutations within $\mathrm{N}$-terminal repeats of the Notch3 receptor awaits further studies.

The broad clinical spectrum of CADASIL ${ }^{2,3}$ raises the question of genotype-phenotype correlations. The clinical and MRI phenotype in the two families with the deletions did not diverge from the usual spectrum. Both index cases had a history of stroke. One of them had developed cognitive deficits, the other suffered from migraine with aura. Also, age at onset for single manifestations in these individuals was within the expected range. This adds to previous clinical and neuroimaging evidence indicating, that the Notch 3 genotype has no major influence on the phenotype.,22

In conclusion we have found a number of new mutations including two small in-frame deletions. These deletions are relevant in that they substantiate the pivotal role of an odd number of cysteine residues within EGF-like repeat domains in the pathogenesis of CADASIL. The 3-D models suggest domain misfolding at least for a subset of mutations including the two deletions.

\section{Acknowledgements}

We gratefully acknowledge the collaboration of all CADASIL patients and their families, and the numerous physicians who referred cases. We are further indebted to $\mathrm{Dr} A$ Joutel and Dr E Tournier-Lasserve (Paris) who provided us with the primer sequences prior to their publication. This work was supported by the Deutsche Forschungsgemeinschaft (Di 722/I-1). Part of this work are part of the thesis of $\mathrm{H}$ Ludwig (in preparation).

Accession numbers and URLs for data in this article are as follows: GenBank, http://www.ncbi.nlm.nih.gov/Entrez/nucleotide.htlm (Notch3 CDNA [U97669]). Online Mendelian inheritance in man (OMIM), http://www3.ncbi.nlm.nih.gov/htbin-post/Omim/ dispmim?125310 (for CADASIL [MIM 125310]).

\section{References}

1 Tournier-Lasserve E, Joutel A et al: Cerebral autosomal dominant arteriopathy with subcortical infarcts and leukoencephalopathy maps on chromosome19q12. Nat Genet 1993; 3: 256-259.

2 Chabriat $H$, Vahedi $K$, Iba Zizen MT et al: Clinical spectrum of CADASI L: a study of 7 families. Lancet 1995; 346: 934-939.

3 Dichgans M, Mayer M, Uttner I et al: The phenotypic spectrum of CADASIL: clinical findings in 102 cases. Ann Neurol 1998; 44: 731-739.

4 Chabriat $\mathrm{H}$, Levy $\mathrm{C}$, Taillia $\mathrm{H}$ et al: Patterns of MRI lesions in CADASI L. Neurology 1998; 51: 452-457.

5 Ruchoux MM, Maurage CA: CADASIL: cerebral autosomal dominant arteriopathy with subcortical infarcts and leukoencephalopathy. J Neuropathol Exp Neurol 1997; 56: 947-964.

6 Joutel A, Corpechot C, Ducros A et al: Notch3 mutations in CADASI L, a hereditary adult-onset condition causing stroke and dementia. Nature 1996; 383: 707-710.

7 Artavanis-Tsakonas S, Matsuno K, Fortini ME: Notch signaling. Science 1995; 268: 225-232.

8 Weinmaster G: The ins and outs of Notch signaling. Mol Cell Neurosci 1997; 9: 91-102.

9 Doolittle RF, Feng DF, Johnson MS: Computer-based characterization of epidermal growth factor precursor. Nature 1984; 307: 558-560.

10 Campbell ID, Bork P: Epidermal growth factor-like modules. Curr Opin Struct Biol 1993; 3: 385-392. 
11 Moy FJ, Li YC, Rauenbuehler P, Winkler ME, Scheraga HA, Montelione GT: Solution structure of human type-alpha transforming growth factor determined by heteronuclear NMR spectroscopy and refined by energy minimization with restraints. Biochemistry 1993; 32: 7334-7353.

12 Nagata K, Kohda D, Hatanaka $\mathrm{H}$ et al: Solution structure of the epidermal growth factor-like domain of heregulin-alpha, a ligand for P180erbB-4. EMBO | 1994; 13: 3517-3523.

13 Graves BJ, Crowther RL, Chandran C, Rumberger JM, Li S, Huang KS, Presky DH, Familletti PC, Wolitzky BA, Burns DK: Insight into E-selectin/ligand interaction from the crystal structure and mutagenesis of the lec/EGF domains. Nature 1994; 367: 532-538.

14 Hansen AP, Petros AM, Meadows RP, Fesik SW: Backbone dynamics of a two-domain protein: $15 \mathrm{~N}$ relaxation studies of the aminoterminal fragment of urokinase-type plasminogen activator. Biochemistry 1994; 33: 15418-15424.

15 Smith BO, Downing AK, Driscoll PC, Dudgeon TJ, Campbell ID: The solution structure and backbone dynamics of the fibronectin typel and epidermal growth factor-like pair of molecules of tissue plasminogen activator. Structure 1995; 3: 823-833.

16 Downing AK, Knott V, Werner JM, Cardy CM, Campbell ID, Handford PA: Solution structure of a pair of calcium-binding epidermal growth factor-like domains: implications for the Marfan syndrome and other genetic disorders. Cell 1996; 85: 597-605.

17 Joutel $A$, Vahedi $K$, Corpechot $C$ et al: Strong clustering and stereotyped nature of Notch3 mutations in CADASIL patients. Lancet 1997; 350: 1511-1515.

18 Baudrimont M, Dubas F, Joutel A, Tournier-Lasserve E, Bousser MG: Autosomal dominant leukoencephalopathy and subcortical ischemic stroke. A clinicopathological study. Stroke 1993; 24: 122-125.
19 Ruchoux MM, Chabriat H, Bousser MG, Baudrimont M, TournierLasserve E: Presence of ultrastructural arterial lesions in muscle and skin vessels of patients with CADASIL. Stroke 1994; 25: 2291-2922.

20 Jones TA, Zou YJ, Kjeel gard M: Improved methods for building protein models in electron-density maps and location of errors in these models. Acta Crystallogr 1991; A 47: 110-119.

21 Krawczak M, Cooper DN: Gene deletions causing human genetic disease: mechanisms of mutagenesis and the role of the local DNA sequence environment. Hum Genet 1991; 86: 425-441.

22 Dichgans $M$, Filippi $M$, Brüning $R$ et al: Quantitative $M R I$ in CADASIL: correlation with disability and cognitive performance. Neurology 1999; 52: 1361-1367.

23 Oberstein SA, Ferrari MD, Bakker E et al: Diagnostic Notch3 sequence analysis in CADASIL: three new mutations in Dutch patients. Dutch CADASIL Research Group. Neurology 1999; 52: 1913-1915.

24 Cooper DN, Antonarakis SE, Krawczak M: The nature and mechanisms of human gene mutation. In: Scriver CR, Beaudet AL, Sly WS, Valle D, eds. The Metabolic and Molecular Bases of Inherited Disease. 7th edn. McGraw-Hill: New York, 1995, pp 259-291.

25 Lardelli M, Dahlstrand J, Lendahl U: The novel Notch homologue mouse N otch 3 lacks specific epidermal growth factor-repeats and is expressed in proliferating neuroepithelium. Mech Dev 1994; 46: 123-136.

26 Evans SV: SETOR: hardware lighted three dimensional solid model representations of macromolecules. J Mol Graph 1990; 11: 134-138. 